This item was submitted to Loughborough's Institutional Repository (https://dspace.lboro.ac.uk/) by the author and is made available under the following Creative Commons Licence conditions.

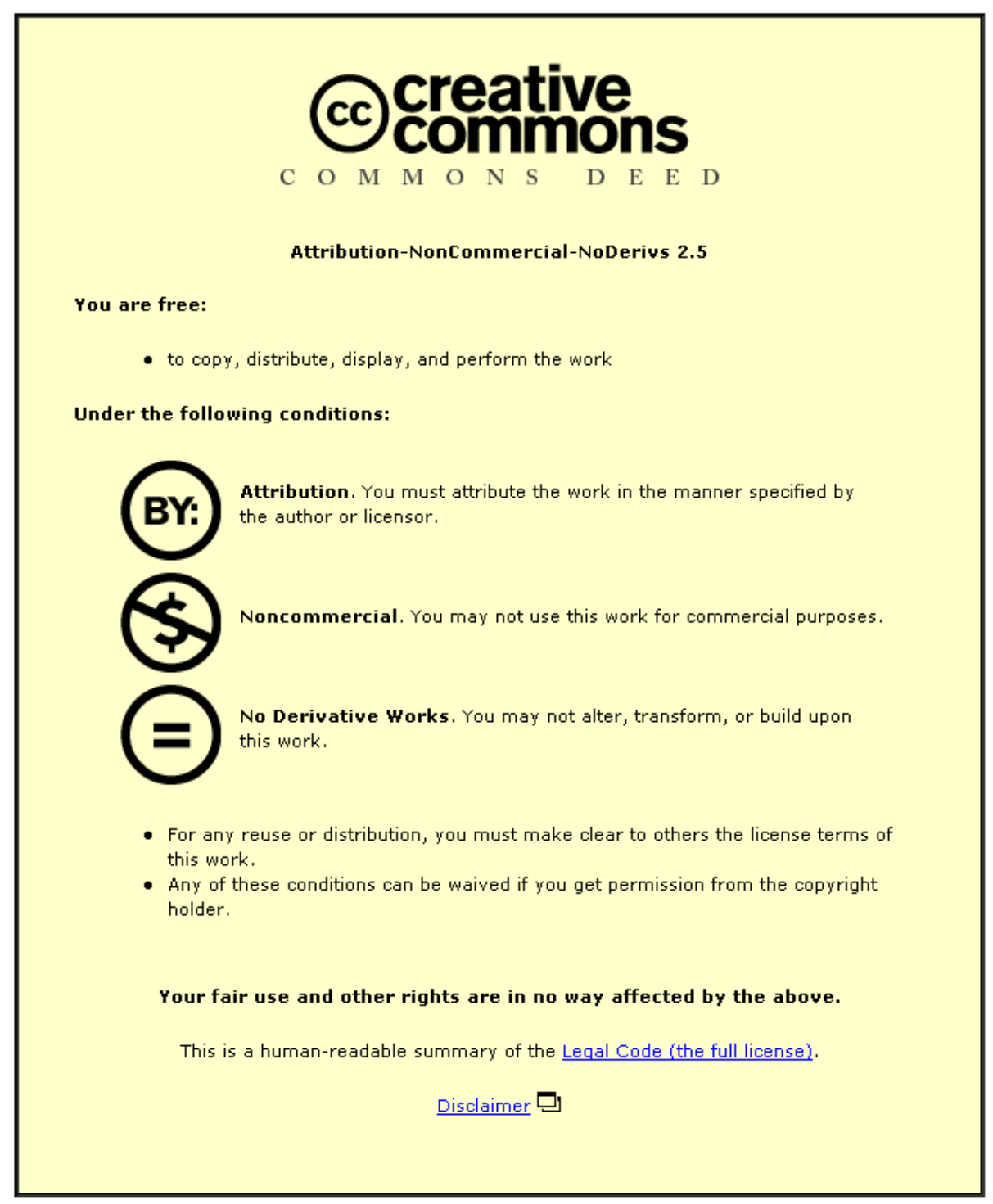

For the full text of this licence, please go to: http://creativecommons.org/licenses/by-nc-nd/2.5/ 


\title{
Plasma stability control using dielectric barriers in radio-frequency atmospheric pressure glow discharges
}

\author{
J. J. Shi, D. W. Liu, and M. G. Kong ${ }^{\text {a) }}$ \\ Department of Electronic and Electrical Engineering, Loughborough University, Loughborough, \\ Leicestershire LE11 3TU, United Kingdom
}

(Received 10 April 2006; accepted 3 July 2006; published online 25 August 2006)

\begin{abstract}
It is widely accepted that electrode insulation is unnecessary for generating radio-frequency (rf) atmospheric pressure glow discharges (APGDs). It is also known that rf APGDs with large discharge current are susceptible to the glow-to-arc transition. In this letter, a computational study is presented to demonstrate that dielectric barriers provide an effective control over unlimited current growth and allow rf APGDs to be operated at very high current densities with little danger of the glow-to-arc transition. Characteristics of electrode sheaths are used to show that the stability control is achieved by forcing the plasma-containing electrode unit to acquire positive differential conductivity. (C) 2006 American Institute of Physics. [DOI: 10.1063/1.2338647]
\end{abstract}

Relieved of the need for a vacuum chamber, atmospheric pressure glow discharges (APGDs) offer an unprecedented capability for low-temperature treatment of metallic and dielectric surfaces. ${ }^{1}$ This generic capability has in the last ten years been brought much closer to practical applications such as biological decontamination and open-air surface coating, ${ }^{1-3}$ benefiting from significant advance in the basic APGD understanding. ${ }^{4-11}$ Of the many recent fundamental APGD studies in literature, a key focus has been different APGD modes ${ }^{7,9,12-14}$ and their influence on plasma stability, particularly in radio-frequency (rf) APGDs. This is important, since atmospheric gas discharges are inherently susceptible to the glow-to-arc transition and an adequate plasma stability in APGDs is often attained at the expense of their application efficiency. ${ }^{10,12}$ It is therefore of particular interest to develop techniques to manipulate and enhance the underpinning APGD dynamics for superior plasma stability. This letter reports a computational study of an unconventional rf APGD that employs dielectrically insulated electrodes to improve its plasma stability. The resulting APGD is effectively a dielectric-barrier discharge (DBD) and is hereafter referred to as rf DBD. As a reference and to avoid any confusion, we refer to the conventional radio-frequency APGD with naked electrodes as rf APGD. In the past, dielectric insulation of electrodes has been widely believed to be necessary for APGD at kilohertz but not at megahertz. ${ }^{4-12}$

Our study is based on a one-dimensional, self-consistent, and continuum model originally developed for rf APGD that has achieved excellent agreement with experimental data. ${ }^{12}$ We consider rf DBD in atmospheric helium. To focus on main plasma characteristics, we consider pure helium as the background gas and ignore the effects of impurity gases. The numerical model includes six plasma species, namely, the electron $e$, helium ion $\mathrm{He}^{+}$, excited helium atom $\mathrm{He}^{*}$, dimer helium ion $\mathrm{He}_{2}^{+}$, excited dimer atom $\mathrm{He}_{2}^{*}$, and background helium atoms He. Relevant reaction mechanisms and transport coefficients are the same as those used in the literature. ${ }^{12,15}$ Rates for reactions involving electrons are expressed in terms of the electron mean energy rather than the

\footnotetext{
a) Author to whom correspondence should be addressed; electronic mail:
} m.g.kong@lboro.ac.uk local electric field to enable an accurate description of the electrode sheath region. ${ }^{11,12,15}$ Both electrodes are covered by a dielectric layer, each with a relative permittivity of 8 and a thickness of $1 \mathrm{~mm}$. The displacement current through each dielectric layer is calculated from

$$
I=C_{d} \frac{\partial V_{m}}{\partial t} .
$$

Here, $C_{d}$ is the capacitance of the two dielectric layers in series. $V_{m}$ is the total voltage across the two dielectric layers, and is hereafter known as the memory voltage. Hence the gas voltage $V_{g}$ is the difference between the applied voltage $V_{a}$ and the memory voltage, or

$$
V_{g}(t)=V_{a}(t)-V_{m}(t)
$$

We assume that the excitation frequency is $13.56 \mathrm{MHz}$, the gas pressure is 760 Torr, and the gas temperature is constant at $300 \mathrm{~K}$. The gas gap sandwiched between the two dielectrically insulated electrodes is fixed at $2.4 \mathrm{~mm}$.

Figure 1 shows typical traces of the computationally obtained discharge current density and various voltages of the rf DBD. They all exhibit a sinusoidal wave form, with the discharge current density leading the gas voltage, the applied

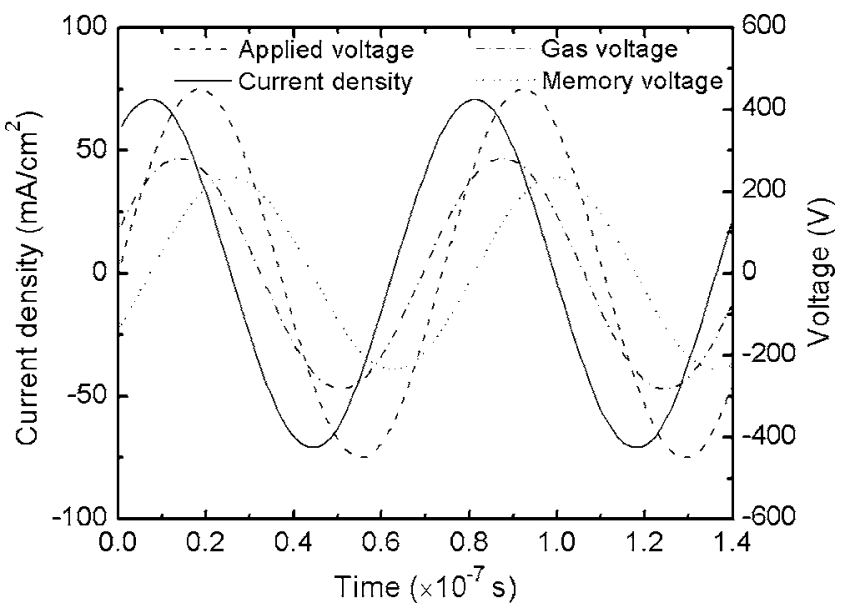

FIG. 1. Typical current density and voltage wave forms of the atmospheric rf DBD. 


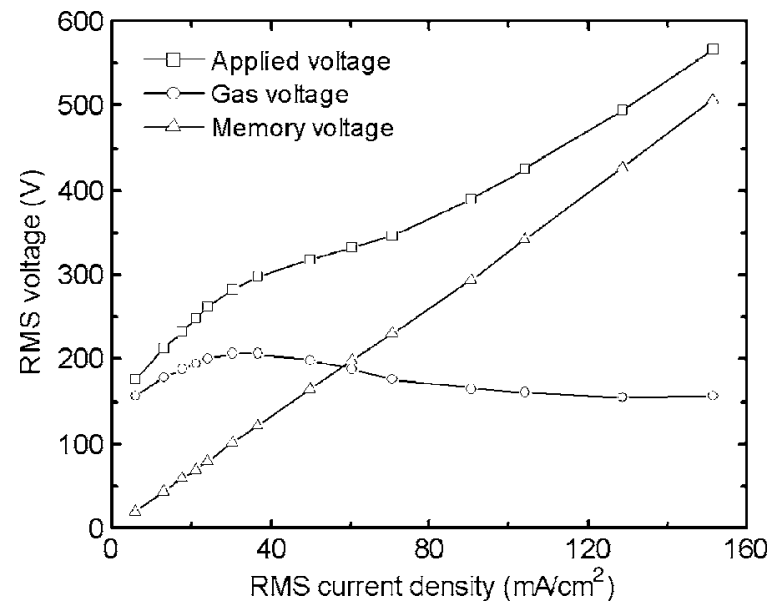

FIG. 2. Current density dependence of the applied voltage (square), the gas voltage (circle), and the memory voltage (triangle).

voltage, and the memory voltage in sequence. This is very different from atmospheric DBD generated at kilohertz frequencies, of which the discharge current and the memory voltage are both distinctively nonsinusoidal. ${ }^{4,5}$ In particular, the discharge current in kilohertz DBD is of very short pulses with its pulse width much smaller than the half-period of the applied voltage. ${ }^{4,5}$ The peak applied voltage of the rf DBD is $450.0 \mathrm{~V}$ in Fig. 1, whereas its peak gas and memory voltages are 281.1 and $234.3 \mathrm{~V}$, respectively. It is of interest to note that the peak discharge density is $70.8 \mathrm{~mA} / \mathrm{cm}^{2}$, markedly greater than what is typical in helium rf APGD. ${ }^{12,15}$

Figure 2 shows the current-voltage characteristics of the rf DBD where the rms values of the applied voltage, the gap voltage, and the memory voltage are plotted as a function of the rms current density. Since the dielectric layers on electrode surfaces are effectively a capacitor in the discharge circuit, their total voltage is linearly proportional to the discharge current as shown in Fig. 2. As the discharge current density in Fig. 2 increases from 5.7 to $151.5 \mathrm{~mA} / \mathrm{cm}^{2}$, the memory voltage goes up from 19.8 to $505.8 \mathrm{~V}$, suggesting a surface impedance of $0.3 \Omega \mathrm{m}^{2}$. This is identical to the total surface reactance of the two dielectric layers at $13.56 \mathrm{MHz}$. Also, the dependence of the gas voltage on the discharge current density is similar to that in conventional rf APGD with naked electrodes. After the gas breakdown and as the current density increases from 5.7 to $36.6 \mathrm{~mA} / \mathrm{cm}^{2}$, the gas voltage first increases from 157.4 to $206.2 \mathrm{~V}$, suggesting that the plasma has a positive differential conductivity. Above 206.2 V however, the gas voltage starts to decrease with increasing current density and so the plasma now has a negative differential conductivity. Therefore, the rf DBD evolves from a region of positive differential conductivity at low current densities to a region of negative differential conductivity at high current densities. This is similar to conventional rf APGD with naked electrodes, ${ }^{7,11-14}$ for which negative differential conductivity has been related to the possibility of significant current growth with decreasing applied voltage. ${ }^{10-12}$ This implies that the discharge current can grow without restriction and with little change in the dissipated $\mathrm{rf}$ power. As a result, a simple technique of restricting the maximum input $\mathrm{rf}$ power is ineffective to control current growth and rf APGDs are vulnerable to the glow-to-arc transition. ${ }^{10}$ So far, the issue of negative differential conductivity has been addressed with avoidance strategies by using, Downloaded 25 Aug 2009 to 158.125.80.71. Redistribution subject

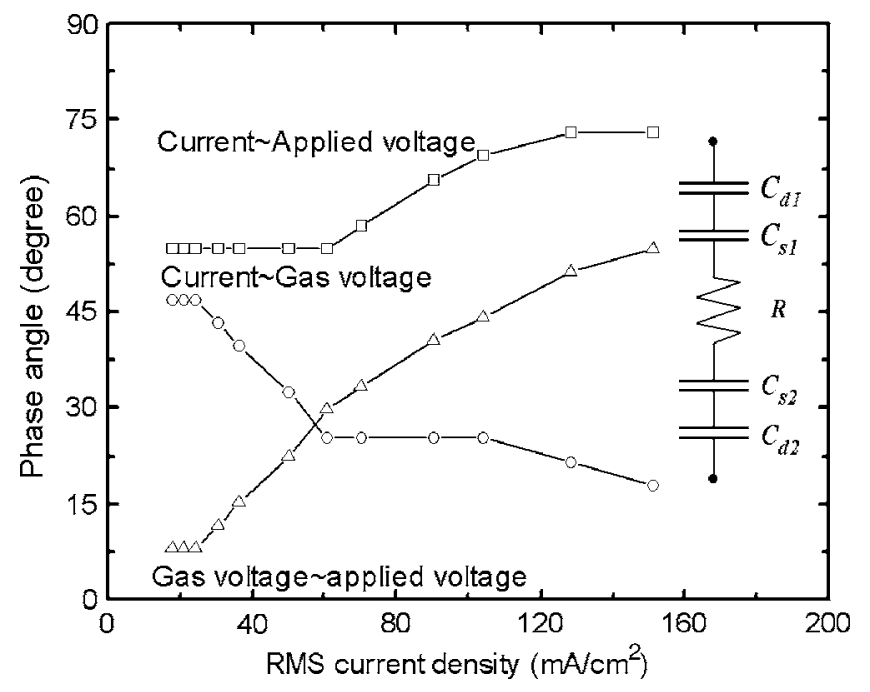

FIG. 3. Current density dependence of the phase angle of the discharge current with the applied voltage (square) and the gas voltage (circle), and that of the phase angle between the gas voltage and the applied voltage (triangle). The equivalent circuit of the $\mathrm{rf} \mathrm{DBD}$ is shown in the insert.

for example, high excitation frequencies ${ }^{10}$ or small electrode gaps. $6,11,13$

Given the direct link of differential conductivity to plasma stability, it is interesting to note that in Fig. 2 the applied voltage always increases with the discharge current density. This suggests that the differential conductivity of the plasma-enclosing electrode unit is always positive even when that of the plasma is negative. So, the rf power fed to the plasma rig can be capped to control effectively an unlimited growth in the discharge current. It is worth emphasizing that this is achieved in the external circuit outside the plasma-generation region. By separating the plasma from the external circuit, the dielectric barriers allow the rf DBD to be operated at very high current densities without the danger of unlimited current growth. Therefore, dielectric barriers are highly desirable for stabilizing rf APGDs at high discharge currents even though they are not essential for generating and sustaining them at low discharge currents.

The above can be further understood by revisiting the $V_{g}-I$ relationship in Fig. 2. At low discharge currents, gas ionization is very weak and the plasma is predominately capacitive. As the discharge current increases, gas ionization becomes significant and the plasma becomes predominately resistive. This can be seen in Fig. 3 where the phase angle between the discharge current and the gas voltage decreases from $46.8^{\circ}$ to $25.2^{\circ}$, evolving from being predominately capacitive to predominately resistive in character. As the discharge current increases, the electrical conductivity of the plasma increases significantly. This is responsible for the plasma acquiring negative differential conductivity after $J$ $\geqslant 36.6 \mathrm{~mA} / \mathrm{cm}^{2}$ in Fig. 2. With the dielectric barriers added to the electrodes, the applied voltage is now dropped onto not only the predominately resistive plasma but also the capacitive barriers. The overall impedance seen by the applied voltage is no longer predominately resistive but now predominately capacitive, as seen in Fig. 3 where the phase angle between the current and the applied voltage increases from $54.9^{\circ}$ to $72.9^{\circ}$ with increasing current density. The surface impedance of the plasma is found to be between 0.1 and $0.6 \Omega \mathrm{m}^{2}$ in the region of negative differential conductivity. Yet the dielectric barriers have a constant surface reactance
to AIP license or copyright; see http://apl.aip.org/apl/copyright.jsp 


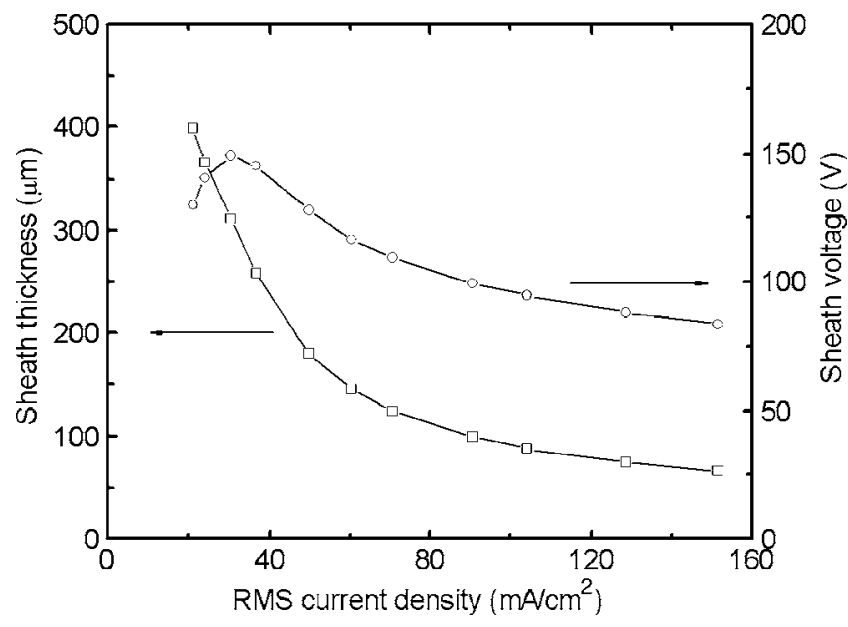

FIG. 4. Current density dependence of the sheath voltage (circle) and the maximum sheath thickness (square).

of $0.3 \Omega \mathrm{m}^{2}$ and therefore can change critically the character of the equivalent load of the plasma-enclosing electrode unit.

Even at very high current densities, it is unusual for rf APGD to become completely resistive..$^{6-15}$ A sheath region depleted of electrons is often formed near the electrodes and it can be realistically modeled as a capacitor. ${ }^{7}$ Therefore the atmospheric rf DBD can be modeled by the equivalent circuit shown in Fig. 3, whereas $C_{d 1}$ and $C_{d 2}$ are capacitances of the two dielectric barriers and $C_{s 1}$ and $C_{s 2}$ are capacitances of the two electrode sheaths. Existence of this electron-depleting sheath region in the atmospheric rf DBD is observed in all cases of our numerical simulation. Figure 4 shows the current density dependence of sheath voltage and sheath thickness. Similar to rf APGD with naked electrodes, the sheath thickness decreases monotonically with increasing current density. At low current densities below $36.6 \mathrm{~mA} / \mathrm{cm}^{2}$, the sheath voltage increases with the current density and the sheath electric field is modest at no more than $9.8 \mathrm{kV} / \mathrm{cm}$. Electrons emitting from the cathode are found numerically to be insufficiently accelerated in the sheath region and their subsequent acceleration in the plasma bulk region is necessary for them to reach the helium ionization energy. ${ }^{12}$ Consequently, gas ionization is likely to be volumetric, occurring both in the sheath region and in the plasma bulk region. ${ }^{11,12}$ The $\mathrm{rf}$ DBD is therefore in the $\alpha$ mode. $^{7-14}$ As the current density increases above $36.6 \mathrm{~mA} / \mathrm{cm}^{2}$, the sheath thickness reduces rapidly and the sheath electric field increases significantly up to $22.0 \mathrm{kV} / \mathrm{cm}$. Most electrons are found numerically to be capable of reaching the helium ionization energy within the sheath region and most gas ionization events are now local- ized near the sheath boundary with the plasma bulk. Therefore in this high current region, the $\mathrm{rf}$ DBD is in the $\gamma$ mode. ${ }^{7-14}$ Contour plots of the ionization rate in the electrode gap (not shown) confirm that gas ionization at current densities below $36.6 \mathrm{~mA} / \mathrm{cm}^{2}$ is volumetric whereas that above $36.6 \mathrm{~mA} / \mathrm{cm}^{2}$ is localized. Therefore, the critical current density of $36.6 \mathrm{~mA} / \mathrm{cm}^{2}$ in Fig. 4 separates the $\alpha$ mode of the rf DBD from its $\gamma$ mode. It also separates the positive differential conductivity from the negative differential conductivity in Fig. 2.

In summary, this study has shown that the dielectric insulation of electrodes can prevent an unlimited growth of the discharge current in radio-frequency APGDs and as such improve their plasma stability significantly. It has been demonstrated that dielectric barriers are effective for stabilizing rf APGD at high discharge currents even though they may not be essential for generating and sustaining rf APGD at low discharge currents. It has also been found that atmospheric $\mathrm{rf}$ DBDs have both the $\alpha$ mode and the $\gamma$ mode, similar to conventional rf APGDs with naked electrodes. Unlike conventional rf APGDs, atmospheric rf DBDs have been shown to be stable in both the $\alpha$ and $\gamma$ modes. As a result, they can be operated at very high current density with much reduced tendency for the glow-to-arc transition.

This work is funded partly by the Department of Health (UK) and partly by the Engineering and Physical Sciences Research Council (UK).

${ }^{1}$ J. R. Roth, Industrial Plasma Engineering (IOP, Philadelphia, 1995), Vol. I, pp. 453-460.

${ }^{2}$ M. Moisan, J. Barbeau, S. Moreau, J. Pelletier, M. Tabrizian, and L. H. Yahia, Int. J. Pharm. 226, 1 (2001).

${ }^{3}$ X. T. Deng, J. J. Shi, G. Shama, and M. G. Kong, Appl. Phys. Lett. 87, 153901 (2005).

${ }^{4}$ I. Radu, R. Bartnikas, and M. R. Wertheimer, IEEE Trans. Plasma Sci. 31, 411 (2003).

${ }^{5}$ M. G. Kong and X. T. Deng, IEEE Trans. Plasma Sci. 31, 7 (2003).

${ }^{6}$ A. P. Yalin, Z. Q. Yu, O. Stan, K. Hoshimiya, A. Rahman, V. K. Surla, and G. J. Collins, Appl. Phys. Lett. 83, 2766 (2003).

${ }^{7}$ J. J. Shi, X. T. Deng, R. Hall, J. D. Punnett, and M. G. Kong, J. Appl. Phys. 94, 6303 (2003).

${ }^{8}$ S. Y. Moon and W. Choe, Appl. Phys. Lett. 84, 188 (2004).

${ }^{9}$ X. Yang, M. Moravej, G. R. Nowling, S. E. Babayan, J. Panelon, J. P. Chang, and R. F. Hicks, Plasma Sources Sci. Technol. 14, 314 (2005).

${ }^{10}$ J. J. Shi and M. G. Kong, Appl. Phys. Lett. 87, 201501 (2005).

${ }^{11}$ J. J. Shi and M. G. Kong, Phys. Rev. Lett. 96, 105009 (2006).

${ }^{12}$ J. J. Shi and M. G. Kong, J. Appl. Phys. 97, 023306 (2005).

${ }^{13}$ R. Rahul, O. Stan, A. Rahman, E. Littlefield, K. Hoshimiya, A. P. Yalin, A. Sharma, A. Pruden, C. A. Moore, Z. Yu, and G. J. Collins, J. Phys. D 38, 1750 (2005).

${ }^{14}$ S. Y. Moon, J. K. Rhee, D. B. Kim, and W. Choe, Phys. Plasmas 13, 033502 (2006).

${ }^{15}$ X. Yuan and L. L. Raja, IEEE Trans. Plasma Sci. 31, 495 (2003). 